\title{
TPH2: A Key Gene Risk Factor and Potential Therapy Target in Depression
}

\author{
Xiaoyan Zhang ${ }^{1, *, \dagger}$, Yiming Wang ${ }^{2, *, \dagger}$ \\ ${ }^{1}$ School of Life Sciences, Sun Yat-sen University, Guangzhou, 510275, China \\ ${ }^{2}$ College of Life Science, Shandong Agricultural University, Shandong, 271018, China \\ $\dagger$ These authors contributed equally.
}

\begin{abstract}
Depression is a world-wide psychological disease and millions of people suffer from it. The illness is basically characterized by low mood with some other diverse manifestations. The mutation in the gene sequence of Tryptophan hydroxylase 2 (TPH2) is one of the several possible causes of the depression, which results in the changed structure and function of $\mathrm{TPH} 2$, and then affects the synthetic process of 5hydroxtrytamine (5-HT), so-called serotonin. The low level of 5-HT contributes to depression eventually, which has been tested by the animal model. This review purports to discuss the emerging relevance between TPH2 and depression as well as signaling pathways mediated by the gene expression, after that some therapeutic methods will be mentioned. It's an urgency to understand the pathogenesis of depression and find more effective therapies, but there still remains a large amount of efforts to make and many mysteries to explore, thereby it is still a long way to go.
\end{abstract}

\section{Introduction}

Depression is a persistent and serious mental illness involving genetic, neurological and cognitive factors [1]. People with depression are often depressed, pessimistic, and even suicidal. Thus, depression not only causes great pain to the patient, but also take their lives, leaving friends and families' heartbroken and lead to a loss of workforce for society. Patients suffering from depression shows some pathological feature in their brains, also some experimental and clinical evidences suggest that neuronal serotonergic and noradrenergic functions of the central nervous system are altered in depressed patients [2-4]. Among these evidences, TPH2 is one of the genes that have been proved significantly related to depression.

Tryptophan hydroxylase (TPH) is an aromatic amino acid hydroxylase, which has been found mainly express in brain stem and gut enterochromaffin cells. In 2003, Walther et al. found a human genomic clone of TPH in neuron, which they called Tryptophan hydroxylase 2 (TPH2) [5]. In mouse, TPH2 mostly express in brain, whereas TPH1 mainly express in periphery [6]. TPH2 contributes to the two-step synthesis of 5hydroxytryptamine (5-HT) as a rate-limiting enzyme. 5HT act as neurocrine, neuromodulator and other physiological roles [7]. It has been shown an association with many emotional disorders, such as depression [8], deficit/hyperactivity disorder and bipolar disorder $[9,10]$. TPH2 takes charge of catalyzing tryptophan into 5-HT, thus it may cause a lack of 5-HT in human's brain if TPH2 were mutant, which contributes to high risk of emotional disorders [6].

In this review, the status quo of depression is introduced, and the role of TPH2 polymorphisms play in depression based on daily researches has been discussed. What's more, the TPH 2 related pathways are also been shown. In the end, this study gathers some representative treatments for depression, and proposes potential therapies targeting molecules from 5-HT system in neurons. These might inspire further study for linkage between TPH 2 and depression, and guide future medical and genetic treatments for depression focusing on TPH2 related pathways as well.

\section{Depression disorder}

Depression is so much normal in our daily life that in accordance with the World Health Organization (WHO), and more than 350 million individuals suffer from the disease. It effects a wide range of people, from the elderly to teenagers no matter what their careers are. The performance of depressive patients is diverse, but all shares black mood. They easily lose interest in everything as well as get anorexial. Disturbances of circadian rhythms is also a cardinal feature [11], which involves in insomnia. A mild depression episode manifests as sadness, anhedonia and a feeling of worthlessness, whereas Major depressive disorder (MDD) is classified by a recurring intention to commit suicide [12]. Suicidal tendency is exceedingly common among the people with MDD [13], almost half of which [14]. This tendency basically derives from a low sense of self-worth [15], making them

\footnotetext{
*Corresponding author: zhangxy329@mail2.sysu.edu.cn, WangYM2021415@163.com
} 
disapprove of themselves and occasionally feel guilt. In general, depression is a dangerous psychological sickness and heavily detrimental to people's mental and physical health.

\section{Relationship between TPH2 and depression}

\subsection{TPH2 gene expression}

TPH2 gene locates on chromosome 11p14-15.3 in human, and encodes 490 amino acids protein, principally express in human brain. Until now, 21639 single nucleotide polymorphisms (SNPs) of TPH2 has been discovered, 29 of which found related to depression according to GeneCards database (March 2021). Some of the mutations can cause great damage to the structure and function by affecting the catalytic and oligomerization domains of the protein, which change stability and solubility [16]. Through ten algorithms, researchers predicted the functional and stability effects of the TPH2 mutations and found the mutation happens at catalytic domain cause more damage to protein structure and function [17].

\subsection{Emerging relevance of TPH2 in depression}

Several reviews about relationship between TPH2 polymorphisms and psychiatirc disorders also reveal the participation of TPH2 in depression. A meta-analysis of association studies between TPH2 SNPs and various psychiatric disorders shows, six SNPs are significantly associated with depression (Table 1) and also other kinds of mood disorder [18]. Whereas, research found rs4565946 C/T genotype related to decreased risk of lateonset depression [19].
Table1. Pooled results of odds ratios (ORs) for allele frequencies per SNP [18]

\begin{tabular}{|c|c|c|c|c|c|}
\hline $\begin{array}{l}\text { SNP } \\
\text { allele }\end{array}$ & contrast & studies & patients & controls & $\begin{array}{l}\text { OR }(95 \% \\
\mathrm{CI})\end{array}$ \\
\hline $\begin{array}{l}\text { rs457062 } \\
5\end{array}$ & $\mathrm{G}(v s \mathrm{~T})$ & 7 & 1848 & 1906 & $\begin{array}{l}1.13(1.02- \\
1.25) \#\end{array}$ \\
\hline $\begin{array}{l}\text { rs171107 } \\
47\end{array}$ & $\mathrm{G}(v s \mathrm{~A})$ & 5 & 1477 & 1458 & $\begin{array}{l}1.15(1.00- \\
1.31) \#\end{array}$ \\
\hline $\begin{array}{l}\text { rs2 } 217136 \\
3\end{array}$ & $\mathrm{G}(v s \mathrm{~A})$ & 4 & 1474 & 1515 & $\begin{array}{l}1.18(0.96- \\
1.43)_{-}^{*}\end{array}$ \\
\hline $\begin{array}{l}\text { rs } 138649 \\
4\end{array}$ & $\mathrm{G}(v s \mathrm{~A})$ & 6 & 1339 & 2160 & $\begin{array}{l}1.17(0.92- \\
1.48)_{-}^{*}\end{array}$ \\
\hline $\begin{array}{l}\text { rs730511 } \\
5\end{array}$ & $\mathrm{G}(v s \mathrm{~A})$ & 7 & 2617 & 3862 & $\begin{array}{l}1.05(0.97- \\
1.13) \#\end{array}$ \\
\hline $\begin{array}{l}\text { rs } 111789 \\
98\end{array}$ & $\mathrm{G}(v s \mathrm{~A})$ & 2 & 416 & 383 & $\begin{array}{l}1.02(0.74- \\
1.42) \#\end{array}$ \\
\hline
\end{tabular}

CI represents confidence interval.

* Random effects analysis.

\# Fixed effects analysis.

There are other researches showing a connection between TPH2 polymorphisms and alcohol-related suicide as well as major depression [20-22]. A research genotyped 289 patients with major disorder from Chinese Han population, found multiple TPH2 gene alleles interact with negative life events, such as rs11178997 T and rs120074175, and individuals who have at least one of them are susceptible to major disorder8. In the studies of pathology, depressed suicides and alcoholics were found increase in TPH 2 mRNA and its protein expression in human dorsal and median raphe nuclei [23-25].

\subsection{TPH2 related signaling pathways}

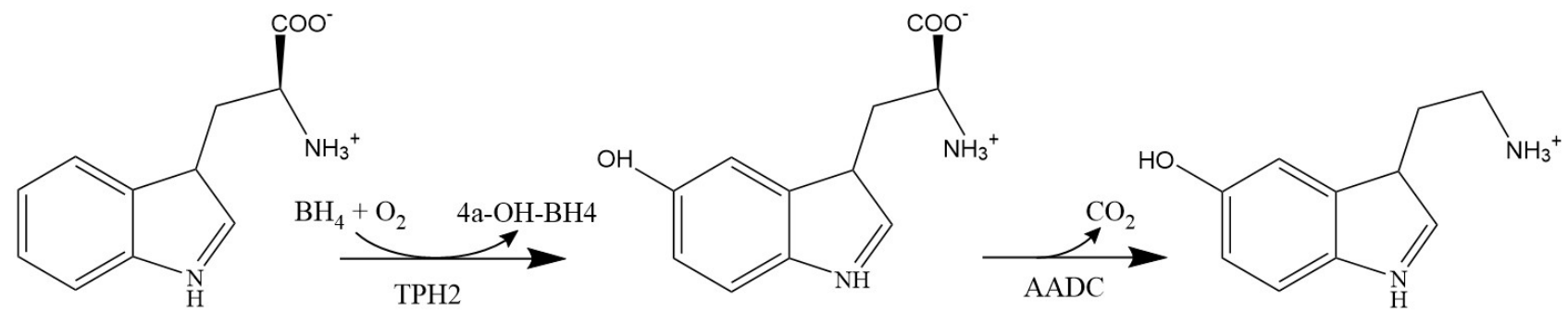

L-tryptophan

5-hydroxy-L-tryptophan

serotonin

Fig 1. Two-step biosynthesis of serotonin.

Tetrahydrobiopterin (BH4) and $\mathrm{O} 2$, together with L-tryptophan, are catalyzed to 4a-hydroxytetrahydrobiopterin (4a-OH-BH4) and 5hydroxytryptophan (5-HTP) by TPH2. Then, 5-HTP is catalyzed to serotonin by ADDC.

The two-step biosynthesis of serotonin is shown in figure 1. Firstly, L-tryptophan (Trp) is metabolized to 5hydroxytryptophan (5-HTP) by rate-limiting enzyme, TPH2. Secondly, 5-HTP is decarboxylated to 5-HT by aromatic amino acid decarboxylase (AADC), which also takes part in the synthesis of dopamine from L-3,4dihydroxyphenylalanine(L-DOPA), tyramine from tyrosine, tryptamine from Trp, showing varied functions and wide distribution [26].
5-HT act as a neurocrine and neuromodulator, and also other physiological roles [7]. There are 2 types of 5-HT receptor in our brain. In a theory, 5-HT1A receptor (5HT1AR) responses to 5-HT and moderate anxiety and stress, and promote patience, called "passive coping", which can only tolerating but not easing psychological pain [27]. While 5-HT1A receptors (5-HT2ARs) gives human flexibility, to deal with psychological pain, called "active coping" [28]. 5-HT1AR can be enhanced by selective serotonin reuptake inhibitors, and 5-HT2AR can 
be enhanced by 5-HT2AR-agonist psychedelics. In TPH2 loss-of-function mice, basal and stimulated levels of extracellular 5-HT reduced, and 5-HT2AR level increased [29]. TPH2 gene and 5-HT2AR gene are considered as potential risk genes for depression. Study shows a potential interaction between TPH2 and 5-HT2AR, which may influence the susceptibility to depression8. Research shows TPH2 mRNA expression is related to changes in serotonergic, glutamatergic and endocannabinoid neurotransmission systems [30]. Thus, TPH2 expression level is affected by multiple messenger systems in relation to presynaptic and/or postsynaptic feedback control of serotonin synthesis.

In 5-HT neurons, 5-HT is stored in vesicles, and released from presynaptic neuron when it's activated (Figure 2). Then 5-HT receptors at postsynaptic neuron membrane conbine with 5-HT and propagate signaling. The serotonin transporter (SERT) takes responsibility in recycling 5-HT back into presynaptic neurons and maintain extracellular levels of 5-HT. Whereas, SERT is also the target of selective serotonin reuptake inhibitor (SSRI) antidepressants, and its reuptake function can be constitutively altered by polymorphisms. If SERT loss of function, 5-HT would be predominantly removed by alternative mechanisms, including uptake through secondary transporters such as organic cation transporters, plasma membrane monoamine transporter, which are expressed on non-serotonergic neurons, astrocytes and serotonergic neurons, then most of transported 5-HT is degraded by monoamine oxidase (MAO) rather than recycled, which will lead to increase in Trp and TPH2 demand, to synthesize more 5-HT. But 5-HT deficient neurons still retain their electrophysiological properties when TPH2 loss of function [31].

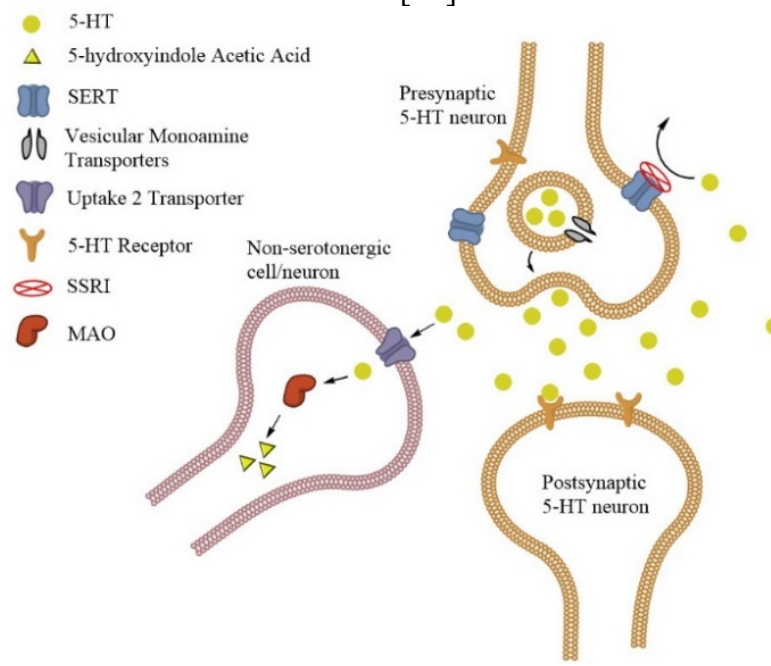

Fig 2. 5-HT signaling, uptake and degradation [31]. $5-\mathrm{HT}$ is stored in vesicles of presynaptic 5-HT neuron, and released after activation, and combine with 5-HT receptor on postsynaptic 5-HT neuron membrane to propagate signaling. 5HT will be reuptaked back into 5-HT neurons by SERT, or into non-serotonergic cells/neurons by uptake 2 transporter. SSRI can inhibit combination of SERT and 5-HT.

Level of TPH2 expression can also affect other neural systems. Study shows TPH2 reduction lead to deficiency of 5-HT metabolism, and coupled with acute stress, they can immediately influence NE and dopamine systems
[32]. Serotonergic and noradrenergic systems have coregulation involved in behavior and psychopathology [33].

\subsection{Experiments in rodent model}

There are also many findings in rodent models found linkage between TPH2 polymorphisms and emotional disorders. Research of TPH2 loss-of-function mice seems reflects chronic, endogenous central nervous system (CNS) 5-HT deficiency [29]. Also, researches on TPH2 knockout rats or mice show a decreased one of the 5HT receptors, 5-HT1AR sensitivity [34,35]. There's experiment shows the reduction of serotonin degradation in TPH2-reduced mice might alleviate the impact on emotional behaviour [36]. But still, another experiment shows the vulnerability to stress-related psychiatric disorders in TPH2-deficient mice still exist in view of environment factors [37]. It couldn't be ignored that the environment impact on whether TPH2 loss-of-function generates depression and other emotional disorder. For example, a research shows mice carrying the $1473 \mathrm{G}$ allele in TPH2 shows similar behavior with normal mice including depression-like behaviors [38], meanwhile another experiment shows $1473 \mathrm{G}$ polymorphism involved in the regulation of the reaction to emotional stress in mice [9]. Thus, the polymorphism of TPH2 increase the risk of depression seems only when individuals facing environmental stress.

In conclusion, various kinds of TPH2 polymorphisms influence 5-HT function, thereby shows impact on emotional disorders with the presence of environmental stress.

\section{Treatments for depression}

\subsection{Psychotherapy}

Psychotherapy requires the psychologist to help patients establish confidence to overcome the disease for the mental regulations [39]. The psychological counseling from them really has a great help for the patients, even for some incurable ones [40]. The cognitive behaviour therapy, one of the most commonly used means of psychotherapy, suggests that depression is countered efficiently by the positive emotion [41,42]. Moreover, music evokes motions by modulating activities in the specific structure of the brain [43]. Interestingly, forestbased activities can also do patients a great favour [44]. These two methods inspire us sunlight and other substances that affect the ambience may also have effects on curing. For example, living under the dull glow aggravates the state of the illness while an open space alleviates that.

\subsection{Pharmacotherapy}

Medication is regarded as an extremely effective way [39]. When choosing antidepressant, it is necessary to comprehensively consider the patients' age, symptoms, physical condition, drug tolerance and follow the 
principle of individualized rational medicine that varies from person to person. The antidepressant medicines are all based on the monoamine hypothesis, revealing depression is caused by the insufficient activity of monoaminergic neurons [45]. By looking up relative information, there is no medicine acts directly on the TPH2, but do have medicines regulating TPH2 related pathway. The most commonly used medicine is SSRI $[46,47]$, which could inhibit the reuptake of 5-HT by the presynaptic membrane and increase the level of 5-HT in the synaptic cleft. So SSRI achieves the purpose of improving patients' depression by increasing the concentration of 5-HT that is originally decreased by the mutant TPH2 $[48,49]$, and an experiment on mouse model has also verified it [50]. However, SSRI has adverse effects [51], like nausea, diarrhea, fatigue or somnolence, but fortunately none of them is serious. Another one is Serotonin-Norepinephrine Reuptake Inhibitor (SNRI), which has similar function as SSRI [52]. Since it has the ability to elevate the concentration of dopamine in prefrontal cortex (PFC), it results in a faster antidepressant effect than SSRI [53]. In conclusion, increasing the level of transmitter is the end result of these medications. The common negative effect of such medicines is slow onset of action, that is, it begins to take effect two to six weeks after taking the drug [54].

\subsection{Physiotherapy}

The physiotherapy gives rise to an excellent performance especially for major depressive disorder55, including repetitive transcranial magnetic stimulation (rTMS) and modified electroconvulsive therapy (MECT). rTMS is used to change and regulate the cortical activity after the stimulation period by repeated current, which makes it a promising approach for the treatment of neurological and psychiatric disorders [55,56]. Based on researches [57], after long-term rTMS treatment, $\beta$-adrenergic receptors and 5-HT receptors in the prefrontal region would increase. Besides, rats model indicates rTMS can induce neuroplasticity [58]. MECT is a safe induction by using means of pulsed current stimulation to improve the physiological state of patients, and regulating the release of transmitters through acting on the central nervous region of the brain for the purpose of balancing the neural mechanism of the brain [59-61]. Previous animal studies have shown an enhanced sensitivity of 5-HT receptors after MECT, which indicates an increasing availability in treating depression [62]. In general, these two methods increase the binding of 5-HT to its receptors, in order to alleviate depression symptoms.

\subsection{Gene Therapy}

Gene therapy is a fresh tool but with some treatments that have been proved workable, including muscular dystrophies and ophthalmologic disorder [63]. As to neurological disorders, the complexity of the central nervous system (CNS) and blood brain barrier makes new drugs hard to develop while gene therapy could overcome these issues. But it is still facing the problems of vector delivery and targeting specific cells in CNS [64]. Nowadays, intergrating (LV) and nonintegrating (AAV) vectors have been successfully applied to aromatic Lamino acid decarboxylase (AADC), providing us with great examples to edit TPH2 gene of depressive patients $[65,66]$. Later the transposon system springs up while it is found to have the ability to deliver relatively larger therapeutic genes than viral vectors [67]. RNA interference is also a perhaps option. On the one hand, an experiment shows the direct infusion of lipopolymeric nanoparticle siRNAs to brain tumors effectively impedes brain tumor growth in mouse [68], so it indicates siRNAs can be transferred into human's brain. On the other hand, siRNAs targeting 5-HT uptake 2 transporters, SERT or MAO enzymes on non-serotonergic neurons' membranes, can innhibit 5-HT degradation [69]. According to these researches, gene therapy for depression disorder is quite feasible.

\section{Conclusion}

This article clarified the significant connection between TPH2 polymorphisms and depression by summarizing experiments of animal models and statistics in human populations. TPH2 polymorphisms can influence 5-HT function, which might lead to emotional disorders including depression, but with the presence of environmental stress. TPH2 expression level is affected by multiple messenger systems in relation to presynaptic and/or postsynaptic feedback control of 5-HT synthesis, such as 5-HT2AR. Meanwhile, level of TPH2 expression can also affect NE and dopamine systems, but the mechanism hasn't clear yet, hence following researches are required to reveal the truth.

Depression is a mood disorder greatly influenced by environment factors, hence, different experimental conditions that are easily ignored might lead to different conclusions, end up confusing the rest of scientists. Moreover, TPH 2 dependent pathway is not the only genetic factor causing the depression, such as brainderived neurotrophic factor (BDNF), so it is necessary to take other genetic factors into account.

As to therapy for depression, psychotherapy focuses on the contact between people and surroundings. Among the pharmacotherapy, SSRI and SNRI are first-line medicines with the function of increasing targeted transmitters' level, but they cannot get rid of certain sideeffects. Physiotherapy including rTMS and MECT promote the binding of 5-HT with its receptors to some extent. Although gene therapy for depression has not been developed yet, it is practical since many clinical trials for neurological disorders have been proved successfully, but still a long-term goal.

Hopefully, this review would give you a better understanding of TPH 2 function in depression and inspire further studies for relationship between TPH2 and depression. In the end, this paper strongly recommend innovation in future medical and genetic treatments for depression focusing on TPH2 related pathways. 


\section{References}

1. Nobis A, Zalewski D, Waszkiewicz N. Peripheral Markers of Depression. J Clin Med. 2020;9(12):3793.

2. Nemeroff CB. Recent advances in the neurobiology of depression. Psychopharmacology bulletin. 2002;36 Suppl 2:6-23.

3. Sullivan PF, Neale MC, Kendler KS. Genetic epidemiology of major depression: review and metaanalysis. The American journal of psychiatry. 2000;157(10):1552-62.

4. Hao Y, Ge H, Sun M, Gao Y. Selecting an Appropriate Animal Model of Depression. Int J Mol Sci. 2019;20(19):4827.

5. Walther DJ, Peter J, Bashammakh S et al. Synthesis of serotonin by a second tryptophan hydroxylase isoform. Science (New York, N.Y.). 2003;299(5603):76

6. Zhang X, Beaulieu J, Sotnikova TD, Gainetdinov RR, Caron MG. Tryptophan hydroxylase-2 controls brain serotonin synthesis. Science (New York, N.Y.). 2004;305(5681):217

7. Schoenichen C, Bode C, Duerschmied D. Role of platelet serotonin in innate immune cell recruitment. Frontiers in bioscience (Landmark edition). 2019;24:514-526

8. Ma J, Xiao H, Yang Y et al. Interaction of tryptophan hydroxylase 2 gene and life events in susceptibility to major depression in a Chinese Han population. J Affect Disorders. 2015;188:304-9

9. McKinney JA, Turel B, Winge I, Knappskog PM, Haavik J. Functional properties of missense variants of human tryptophan hydroxylase 2. Hum Mutat. 2009;30(5):787-94

10. Cichon S, Winge I, Mattheisen M et al. Brain-specific tryptophan hydroxylase 2 (TPH2): a functional Pro206Ser substitution and variation in the 5'-region are associated with bipolar affective disorder. Hum Mol Genet. 2008;17(1):87-97

11. Chi S, Lee MS. Personalized Medicine Using Neuroimmunological Biomarkers in Depressive Disorders. J Pers Med. 2021;11(2):114.

12. Belmaker RH, Agam G. Major depressive disorder. The New England journal of medicine. 2008;358(1):55-68

13. Ma K, Zhang H, Baloch Z. Pathogenetic and Therapeutic Applications of Tumor Necrosis Factor$\alpha$ (TNF- $\alpha$ ) in Major Depressive Disorder: A Systematic Review. Int J Mol Sci. 2016;17(5):733.

14. Nobis A, Zalewski D, Waszkiewicz N. Peripheral Markers of Depression. J Clin Med. 2020;9(12):3793.

15. Matsuo K, Harada K, Fujita $Y$, et al. Distinctive Neuroanatomical Substrates for Depression in Bipolar Disorder versus Major Depressive Disorder. Cereb Cortex. 2019;29(1):202-214.

16. McKinney JA, Turel B, Winge I, Knappskog PM, Haavik J. Functional properties of missense variants of human tryptophan hydroxylase 2. Hum Mutat. 2009;30(5):787-94

17. Pereira GRC, Tavares GDB, de Freitas MC, De Mesquita JF. In silico analysis of the tryptophan hydroxylase 2 (TPH2) protein variants related to psychiatric disorders. Plos One. 2020;15(3):e0229730

18. Ottenhof KW, Sild M, Lévesque ML, Ruhé HG, Booij L. TPH2 polymorphisms across the spectrum of psychiatric morbidity: A systematic review and meta-analysis. Neurosci Biobehav R. 2018;92:29-42

19. Pereira PDA, Romano-Silva MA, Bicalho MAC et al. Association between tryptophan hydroxylase-2 gene and late-onset depression. The American journal of geriatric psychiatry : official journal of the American Association for Geriatric Psychiatry. 2011;19(9):825-9

20. Zill P, Büttner A, Eisenmenger $W$ et al. Single nucleotide polymorphism and haplotype analysis of a novel tryptophan hydroxylase isoform (TPH2) gene in suicide victims. Biol Psychiat. 2004;56(8):581-6

21. Zupanc T, Pregelj P, Paska AV. Tryptophan hydroxylase 2 (TPH 2) single nucleotide polymorphisms, suicide, and alcohol-related suicide. Psychiat Danub. 2013;25 Suppl 2:S332-6

22. Zupanc T, Pregelj P, Tomori M, Komel R, Paska AV. TPH2 polymorphisms and alcohol-related suicide. Neurosci Lett. 2011;490(1):78-81

23. Bach H, Arango V, Kassir SA et al. Alcoholics have more tryptophan hydroxylase $2 \mathrm{mRNA}$ and protein in the dorsal and median raphe nuclei. Alcoholism, clinical and experimental research. 2014;38(7):1894901

24. Bach-Mizrachi H, Underwood MD, Tin A et al. Elevated expression of tryptophan hydroxylase-2 mRNA at the neuronal level in the dorsal and median raphe nuclei of depressed suicides. Mol Psychiatr. 2008;13(5):507-13, 465

25. Bach-Mizrachi H, Underwood MD, Kassir SA et al. Neuronal tryptophan hydroxylase mRNA expression in the human dorsal and median raphe nuclei: major depression and suicide. Neuropsychopharmacology : official publication of the American College of Neuropsychopharmacology. 2006;31(4):814-24

26. Mosienko V, Beis D, Pasqualetti M et al. Life without brain serotonin: reevaluation of serotonin function with mice deficient in brain serotonin synthesis. Behav Brain Res. 2015;277:78-88

27. Deakin J. The origins of '5-HT and mechanisms of defence' by Deakin and Graeff: a personal perspective. Journal of psychopharmacology (Oxford, England). 2013;27(12):1084-9

28. Carhart-Harris RL, Nutt DJ. Serotonin and brain function: a tale of two receptors. Journal of psychopharmacology (Oxford, England). 2017;31(9):1091-1120

29. Jacobsen JPR, Siesser WB, Sachs BD et al. Deficient serotonin neurotransmission and depression-like 
serotonin biomarker alterations in tryptophan hydroxylase 2 (Tph2) loss-of-function mice. Mol Psychiatr. 2012;17(7):694-704

30. Jahanshahi A, Le Maitre E, Temel Y et al. Altered expression of neuronal tryptophan hydroxylase-2 mRNA in the dorsal and median raphe nuclei of three genetically modified mouse models relevant to depression and anxiety. $\mathrm{J}$ Chem Neuroanat. 2011;41(4):227-33

31. Garbarino VR, Gilman TL, Daws LC, Gould GG. Extreme enhancement or depletion of serotonin transporter function and serotonin availability in autism spectrum disorder. Pharmacol Res. 2019;140:85-99

32. Aboagye B, Weber T, Merdian HL et al. Serotonin deficiency induced after brain maturation rescues consequences of early life adversity. Sci Rep-Uk. 2021;11(1):5368-5368

33. Gutknecht L, Araragi N, Merker S et al. Impacts of brain serotonin deficiency following $\mathrm{Tph} 2$ inactivation on development and raphe neuron serotonergic specification. Plos One. 2012;7(8):e43157-e43157

34. Peeters DGA, de Boer SF, Terneusen A et al. Enhanced aggressive phenotype of Tph2 knockout rats is associated with diminished 5-HT(1A) receptor sensitivity. Neuropharmacology. 2019;153:134-141

35. Mlinar B, Montalbano A, Waider J, Lesch K, Corradetti R. Increased functional coupling of 5HT(1A) autoreceptors to GIRK channels in Tph2(-/-) mice. European neuropsychopharmacology : the journal of the European College of Neuropsychopharmacology. 2017;27(12):1258-1267

36. Mosienko V, Matthes $\mathrm{S}$, Hirth $\mathrm{N}$ et al. Adaptive changes in serotonin metabolism preserve normal behavior in mice with reduced TPH2 activity. Neuropharmacology. 2014;85:73-80

37. Lieb MW, Weidner M, Arnold MR et al. Effects of maternal separation on serotonergic systems in the dorsal and median raphe nuclei of adult male Tph2deficient mice. Behav Brain Res. 2019;373:112086

38. Koshimizu H, Hirata N, Takao $\mathrm{K}$ et al. Comprehensive behavioral analysis and quantification of brain free amino acids of C57BL/6J congenic mice carrying the $1473 \mathrm{G}$ allele in tryptophan hydroxylase-2. Neuropsychopharmacology reports. 2019;39(1):5660

39. Bernaras E, Jaureguizar J, Garaigordobil M. Child and Adolescent Depression: A Review of Theories, Evaluation Instruments, Prevention Programs, and Treatments. Front Psychol. 2019;10:543.

40. Akechi T, Okuyama T, Onishi J, Morita T, Furukawa TA. WITHDRAWN: Psychotherapy for depression among incurable cancer patients. Cochrane Database Syst Rev. 2018;11(11):CD005537.

41. Nicole Geschwind et al. Positive cognitive behavior therapy in the treatment of depression: A randomized order within-subject comparison with traditional cognitive behavior therapy[J]. Behaviour Research and Therapy, 2019, $116: 119-130$.

42. Health Quality Ontario . Psychotherapy for Major Depressive Disorder and Generalized Anxiety Disorder: A Health Technology Assessment. Ont Health Technol Assess Ser. 2017;17(15):1-167.

43. Gadd Shannon and Tak Casey and Bulaj Grzegorz. Developing music streaming as an adjunct digital therapy for depression: A survey study to assess support from key stakeholders[J]. Journal of Affective Disorders Reports, 2020, 2.

44. Rosa Claudio D. et al. Forest therapy can prevent and treat depression: Evidence from meta-analyses[J]. Urban Forestry \& Urban Greening, 2021, 57.

45. Ushakova V M et al. [Molecular Biological Aspects of Depressive Disorders: A Modern View].[J]. Molekuliarnaia biologiia, 2020, 54(5) : 725-749.

46. Yohn CN, Gergues MM, Samuels BA. The role of 5HT receptors in depression. Mol Brain. 2017;10(1):28.

47. Ijaz S, Davies P, Williams CJ, Kessler D, Lewis G, Wiles N. Psychological therapies for treatmentresistant depression in adults. Cochrane Database Syst Rev. 2018;5(5):CD010558.

48. Banzi R, Cusi C, Randazzo C, Sterzi R, Tedesco D, Moja L. Selective serotonin reuptake inhibitors (SSRIs) and serotonin-norepinephrine reuptake inhibitors (SNRIs) for the prevention of tension-type headache in adults. Cochrane Database Syst Rev. 2015;2015(5):CD011681.

49. Dale E, Pehrson AL, Jeyarajah T, et al. Effects of serotonin in the hippocampus: how SSRIs and multimodal antidepressants might regulate pyramidal cell function. CNS Spectr. 2016;21(2):143-161.

50. Narek Israelyan et al. Effects of Serotonin and SlowRelease 5-Hydroxytryptophan on Gastrointestinal Motility in a Mouse Model of Depression[J]. Gastroenterology, 2019, 157(2) : 507-521.

51. Nanjapp Muralidhara Shankarapura et al. Use of Selective Serotonin and Norepinephrine Reuptake Inhibitors (SNRIs) in the treatment of Autism Spectrum Disorder (ASD), Comorbid psychiatric disorders and ASD-associated symptoms: A Clinical Review.[J]. CNS spectrums, 2020: 21-23.

52. Compagner Chad and Lester Corey and Dorsch Michael. Sentiment Analysis of Online Reviews for Selective Serotonin Reuptake Inhibitors and Serotonin-Norepinephrine Reuptake Inhibitors[J]. Pharmacy, 2021, 9(1) : 27-27.

53. Jie Li et al. SNRIs achieve faster antidepressant effects than SSRIs by elevating the concentrations of dopamine in the forebrain[J]. Neuropharmacology, 2020, 177.

54. Roberts Clark and Sahakian Barbara J. and Robbins Trevor W.. Psychological mechanisms and functions of 5-HT and SSRIs in potential therapeutic change: Lessons from the serotonergic modulation of action 
selection, learning, affect, and social cognition[J]. Neuroscience \& Biobehavioral Reviews, 2020, 119 : 138-167.

55. Pink Aimee E. et al. The use of repetitive transcranial magnetic stimulation (rTMS) following traumatic brain injury (TBI): A scoping review[J]. Neuropsychological Rehabilitation, 2021, 31(3) : 479-505.

56. McClintock SM, Reti IM, Carpenter LL, et al. Consensus Recommendations for the Clinical Application of Repetitive Transcranial Magnetic Stimulation (rTMS) in the Treatment of Depression. J Clin Psychiatry. 2018;79(1):16cs10905.

57. Health Quality Ontario. Repetitive Transcranial Magnetic Stimulation for Treatment-Resistant Depression: A Systematic Review and MetaAnalysis of Randomized Controlled Trials. Ont Health Technol Assess Ser. 2016;16(5):1-66.

58. Toledo Roberta Ströher et al. rTMS induces analgesia and modulates neuroinflammation and neuroplasticity in neuropathic pain model rats[J]. Brain Research, 2021, 147427.

59. Lefaucheur J, Andre-obadia N, Antal A et al. Evidence-based guidelines on the therapeutic use of repetitive transcranial magnetic stimulation(rTMS) [J].Clinical Neurophysiology, 2014, 125(11):21502206.

60. Kerner N, Prudic J. Current electroconvulsive therapy practice and research in the geriatric population. Neuropsychiatry (London). 2014;4(1):33-54.

61. Cretaz E, Brunoni AR, Lafer B. Magnetic Seizure Therapy for Unipolar and Bipolar Depression: A Systematic Review. Neural Plast. 2015;2015:521398.

62. Li M, Yao X, Sun L, et al. Effects of Electroconvulsive Therapy on Depression and Its Potential Mechanism. Front Psychol. 2020;11:80.

63. O'Reilly M, Federoff HJ, Fong Y, et al. Gene therapy: charting a future course--summary of a National Institutes of Health Workshop, April 12, 2013. Hum Gene Ther. 2014;25(6):488-497.

64. Kumar SR, Markusic DM, Biswas M, High KA, Herzog RW. Clinical development of gene therapy: results and lessons from recent successes. Mol Ther Methods Clin Dev. 2016;3:16034.

65. Cartier N, Hacein-Bey-Abina S, Bartholomae CC et al. Hematopoietic stem cell gene therapy with a lentiviral vector in X-linked adrenoleukodystrophy. Science (New York, N.Y.). 2009;326(5954):818-23

66. Hwu W, Muramatsu S, Tseng S et al. Gene therapy for aromatic L-amino acid decarboxylase deficiency. Sci Transl Med. 2012;4(134):134ra61

67. Tipanee J, Chai YC, VandenDriessche T, Chuah MK. Preclinical and clinical advances in transposon-based gene therapy. Biosci Rep. 2017;37(6):BSR20160614. Published 2017 Dec 5.

68. Yu D, Khan OF, Suvà ML, et al. Multiplexed RNAi therapy against brain tumor-initiating cells via lipopolymeric nanoparticle infusion delays glioblastoma progression. Proc Natl Acad Sci U S A. 2017;114(30):E6147-E6156.

69. Wu SY, Lopez-Berestein G, Calin GA, Sood AK. RNAi therapies: drugging the undruggable. Sci Transl Med. 2014;6(240):240ps7. 